\title{
Increased miR-132 level is associated with visual memory dysfunction in patients with depression
}

This article was published in the following Dove Press journal:

Neuropsychiatric Disease and Treatment

9 November 2016

Number of times this article has been viewed

\author{
Ye Liu ${ }^{1,2}$ \\ Xiao Yang ${ }^{1,2}$ \\ Liansheng Zhao ${ }^{1,2}$ \\ Jian Zhang ${ }^{1,2}$ \\ Tao $\mathrm{Li}^{1,2}$ \\ Xiaohong $\mathrm{Ma}^{1,2}$ \\ 'Psychiatric Laboratory, Department \\ of Psychiatry, ${ }^{2}$ National Key \\ Laboratory of Biotherapy, West China \\ Hospital, Sichuan University, Chengdu, \\ People's Republic of China
}

Correspondence: Xiaohong Ma Psychiatric Laboratory, Department of Psychiatry, West China Hospital, Sichuan University, Chengdu, Sichuan 6I0064,

People's Republic of China

Tel +862885I640I9

Email maxiaohong@scu.edu.cn
Background: Impaired visual memory seems to be a core feature of depression, while increased microRNA-132 (miR-132) levels have been widely reported in depression patients. The authors aimed to explore the relationship between miR-132 changes and visual memory deficits in unmedicated patients with major depressive disorder (MDD).

Patients and methods: A total of 62 medication-free MDD patients and 73 matched healthy controls (HCs) were tested for miR-132 expression level in peripheral blood using quantitative realtime polymerase chain reaction. We used a computerized neurocognitive task from the Cambridge Neuropsychological Test Automated Battery (CANTAB) - pattern recognition memory (PRM) task - as a measurement of visual memory. The relationship between visual memory, miR-132 expression level, and clinical symptoms was explored in patients with MDD.

Results: Upregulated miR-132 expression levels were seen in MDD patients but not in HCs. Two-sample $t$-tests showed that MDD patients had decreased visual memory, mainly memory delayed compared to that of HCs. Correlation analyses revealed that in MDD patients, increased miR-132 expression levels were significantly correlated with visual memory as measured by the CANTABPRM. Hamilton Rating Scale for Anxiety scores were negatively correlated with PRM - number correct (immediate) and PRM - percent correct (immediate).

Limitations: The main limitations were missing data and lack of follow-up studies.

Conclusion: Our study suggests that increased miR-132 expression levels were associated with visual memory deficits, which may underlie the pathophysiology of MDD. In individuals with depression, immediate visual memory defects were positively correlated with anxiety symptoms.

Keywords: major depressive disorder, miR-132, cognition, memory, CANTAB

\section{Introduction}

Depressive disorders, affecting 121 million people worldwide, are a major public health concern. The prevalence of depression is known to be between $5 \%$ and $19 \%{ }^{1}$ Regarding course of recovery, major depressive disorder (MDD) was historically seen as an episodic disorder, but recent findings have indicated that $50 \%$ of depressed patients experience a relapse within 2 years after their first episode and $80 \%$ experience more than one depressive episode during their life course. ${ }^{2}$ Although depression traditionally is seen as affective disorder in nature, research conducted during the last decades has shown that depression is associated with important disturbance in cognitive functioning, such as memory loss, attention deficit, and executive function impairment. ${ }^{3,4}$ Among them, memory decline was reported by most patients with depression, which seriously affected their social functioning and quality of life. ${ }^{3}$ Numerous studies showed that visual memory impairment is one of the important features of memory defects in depression patients (DPs). ${ }^{5-7}$ 
Despite considerable efforts to underpin the molecular and cellular changes associated with depressive states, a global understanding of the pathophysiology of MDD is still lacking. Recently, some studies particularly focused on the interplay between genetic and environmental factors. Through epigenetic mechanisms, environmental factors can induce changes in gene expression levels that might mediate the onset of a disease without altering the DNA sequence. ${ }^{8,9}$ These mechanisms include histone modification, DNA methylation, and post-transcriptional regulation by non-coding RNAs such as microRNAs (miRNAs). It is now generally accepted that these epigenetic factors may mediate susceptibility to disorders like MDD. ${ }^{10}$ miRNAs are short ( $\sim 22$ nucleotides) RNA molecules that negatively regulate gene expression at the post-transcriptional leve $\mathrm{l}^{11}$ and trigger mRNA degradation and/or translational repression in turn. ${ }^{12,13}$ Each miRNA may target several mRNAs, often in specific locations on their $3^{\prime}$-untranslated region (3'-UTR). More than 1,000 reported human miRNAs (miRbase database ${ }^{14}$ ) are estimated to control the expression of more than half of human genes. ${ }^{15}$ Within the central nervous system (CNS), a recent work has indicated that miRNAs play key roles in a wide range of physiological processes, including neurogenesis, neuronal proliferation, and synaptic plasticity. ${ }^{5}$ The disruption of miRNA expression through genetic deletion of Dicer results in aberrant neuronal development and differentiation, as well as altered morphogenesis and neuronal signaling. ${ }^{5}$ For example, Konopka et $\mathrm{al}^{16}$ demonstrated that conditional deletion of Dicer1 enhanced learning and memory capacity in mice.

A growing number of studies on psychiatric illness have focused on miRNAs in peripheral blood. ${ }^{17-20}$ First, the CNS may exert its influence on the gene expression of peripheral lymphocytes via cytokines, neurotransmitters, or hormones, ${ }^{5}$ which may explain the comparable gene expression levels between the peripheral blood and some CNS tissues. ${ }^{5}$ Meanwhile, this also provides the explanation of alterations in the dopamine transporter in the lymphocytes of psychotic patients. ${ }^{5}$ Second, the peripheral expression levels of some dysregulated genes were also found to be correlated with certain clinical symptoms and neurocognitive functions in patients with depression. ${ }^{5}$ In fact, blood-based miRNAs are easier to access and less intrusive than brain tissues, which is becoming an increasingly important approach to identify clinically applicable biomarkers for MDD.

Altered expressions of microRNA-132 (miR-132) are associated with a series of neurological disorders, such as Alzheimer's disease, ${ }^{21}$ Huntington disease, ${ }^{22,23}$ drug addiction, ${ }^{24}$ and schizophrenia. ${ }^{5}$ Previous studies have also shown that miR-132 is related to proteins or genes, which are important for depression. ${ }^{25}$ For example, a study revealed that miR-132 induced by light in the suprachiasmatic nucleus regulates the expression of period genes, which may play a role in circadian rhythms. ${ }^{26}$ We know that impaired neurogenesis and alteration of circadian rhythms as potential dysregulated pathways may contribute to depressive disorders. Another study revealed that miR132 targets p250GAP, a Rac1 GTPase-activating protein. ${ }^{27}$ By repressing p250GAP levels, miR-132 increases the activity of the actin depolymerizing protein n-cofilin via Rac1-PAK signaling. ${ }^{28}$ This modulation of actin turnover in dendritic spines has been shown to regulate both spine size and spine density. ${ }^{29,30}$ Inhibition of miR-132 in primary hippocampal cultures decreased spine density and size, whereas in vivo overexpression increased spine density. We know that memory function is closely related to the number and density of spine.

Despite these findings, the mechanism of cognitive function defects in MDD is not fully understood till now. In this study, we aimed to explore the possible association between peripheral miR-132 expression level and visual memory defects in patients with MDD.

\section{Patients and methods Participants and blood samples}

Although we included 72 drug naïve patients and 74 healthy controls (HCs), 11 individuals failed to fully complete the CANTAB test for personal reasons and were excluded. The final sample size was 62 patients and $73 \mathrm{HCs}$. All participants were recruited from the Mental Health Center of West China Hospital of Sichuan University. All patients were independently diagnosed by two licensed psychiatrists according to Structured Clinical Interview for DSM-IV Axis I Disorders (SCID), thus excluding patients with concurrent other axis I disorders except major depression. ${ }^{31}$ All patients were assessed immediately after the baseline interview. Disease severity was evaluated using the Hamilton Rating Scale for Depression (HAMD; 17-item version, 1960) and Hamilton Rating Scale for Anxiety (HAMA; 14-item version, 1959). The exclusion criteria included age younger than 18 years or older than 60 years, severe medical diseases, mental retardation, structural brain disorders, movement disorders, and drug abuse. Meanwhile, demographically similar (age-, gender-, and education-matched) healthy volunteers were recruited via advertisements within the local community. The control subjects were also interviewed according to 
the SCID, non-patient edition, ${ }^{32}$ to assure that no one had a current or past history of depression or other axis I disorders. The ethical committee of Sichuan University approved the study and the sample collection procedures. Written informed consent was obtained from all participants.

\section{RNA isolation}

Whole blood (3 $\mathrm{mL}$ ) was collected from each participant using Tempus Blood RNA Tubes (Thermo Fisher Scientific, Waltham, MA, USA; PN: 4342792). Blood was mixed vigorously for at least 10 seconds after collection and immediately frozen at $-80^{\circ} \mathrm{C}$ until use. Total RNAs were isolated using the MagMAX for Stabilized Blood Tubes RNA Isolation Kit (PN: 4451893; Ambion, Carlsbad, CA, USA) according to the manufacturer's protocol. The RNA quality was assayed using NanoDrop ND-2000 (Thermo Fischer Scientific), and integrity was assessed using Bioanalyzer 2100 (Agilent Technologies, Santa Clara, CA, USA).

\section{cDNA synthesis and quantitative real- time polymerase chain reaction ( $q P C R$ )}

Portions (100 ng) of each of the total isolated RNA preparations were reverse transcribed to cDNA using a TUREscript 1st Strand cDNA SYNTHESIS Kit (PC1802; Aidlab, Beijing, People's Republic of China) according to the manufacturer's instructions. The reaction mixture consisted of $2 \mu \mathrm{L} 5 \times$ RT Reaction Mix, $0.8 \mu \mathrm{L}$ gene special primer (GSP) (miR-132 reverse transcription primer), and $100 \mathrm{ng}$ total RNA template in a total volume of $10 \mu \mathrm{L}$. Reverse transcription was performed in GeneAmp PCR System 9700 (Thermo Fisher Scientific) at $42^{\circ} \mathrm{C}$ for $60 \mathrm{~min}$ and $85^{\circ} \mathrm{C}$ for $5 \mathrm{~min}$. The resulting reverse transcription reaction product was immediately stored at $-20^{\circ} \mathrm{C}$ for further analysis. The qPCR reactions were done in real-time cyclers (qTOWER 2.2; Analytik Jena, Jena, Thuringia, Germany). The reaction mixture consisted of $5 \mu \mathrm{L} 2 \times$ SYBR Green Supermix (Aidlab; PC3302), 200 nM miRNA specific primers, $200 \mathrm{nM}$ universal primers, and $0.8 \mu \mathrm{L}$ reverse transcription product; the volume was adjusted to $10 \mu \mathrm{L}$ by $\mathrm{H}_{2} \mathrm{O}$. PCR reactions were initiated with 3 -min incubation at $95^{\circ} \mathrm{C}$, followed by 40 cycles at $95^{\circ} \mathrm{C}$ for $10 \mathrm{~s}$ and at $60^{\circ} \mathrm{C}$ for $60 \mathrm{~s}$. A melting curve was performed at the end of the PCR run over a range of $66^{\circ} \mathrm{C}-99^{\circ} \mathrm{C}$, increasing the temperature stepwise by $0.5^{\circ} \mathrm{C}$ every 2 s. Experiments were performed, as described earlier, in triplicate (Table 1). The relative level of miR-132 was calculated with the comparative $\Delta C_{\mathrm{t}}\left(\Delta \Delta C_{\mathrm{t}}\right)$ method using small RNA U6 as a normalization control. Fold change was calculated using $2^{-\Delta \Delta C_{\mathrm{t}}}$.
Table I Sequence of primers in RT-qPCR

\begin{tabular}{ll}
\hline Name & Sequence 5'-3' $^{\prime}$ \\
\hline U6-F & CTCGCTTCGGCAGCACA \\
U6-R & AACGCTTCACGAATTTGCGT \\
miR-132-RT & GTCGTATCCAGTGCAGGGTCCGAGG \\
miR-132-F & TATTCGCACTGGATACGACCGACCA \\
miR-132-R & GCCCGTAACAGTCTACAGCCAT \\
\hline
\end{tabular}

Abbreviations: $R T-q P C R$, reverse transcription quantitative polymerase chain reaction; miR-I32-RT, microRNA-132 reverse transcription primer; miR-I32-F, microRNA-132 forward primer; miR-132-R, microRNA-132 reverse primer.

\section{Visual memory test}

We used pattern recognition memory (PRM) in the Cambridge Neuropsychological Test Automated Battery (CANTAB) to assess visual memory processing. ${ }^{33}$ CANTAB is a standard, computerized, nonlinguistic, and culturally blind test to assess cognitive dysfunction in various psychiatric disorders 5 in experimental and clinical settings. ${ }^{34}$

The PRM test is designed to measure the capacity of visual processing in a two-choice forced discrimination paradigm. ${ }^{35}$ The participants were presented with a series of visual geometric patterns, one at a time, in the center of the screen. These patterns were designed so that they cannot be easily given verbal labels. In the recognition phase, the participants were required to choose the correct pattern between an already seen pattern and a novel pattern. In this phase, the test patterns were presented in the reverse order to the original order of presentation. This was then repeated with new patterns. The second recognition was given either immediately or after a delay. The immediate mode always ran $\sim 20$ min before the delayed mode.

\section{Statistical analysis}

All statistical analyses were performed using SPSS 21.0 software (IBM Corporation, Armonk, NY, USA) for Mac. We used two-sample $t$-test or chi-square test to compare the demographic data. Student's $t$-test was used to identify significant differences in the relative expression of miR-132 and PRM test scores, respectively, between the healthy participants and patients. The relationship between the expression of miR-132 and visual memory function was examined using Pearson's correlation coefficients. $P \leq 0.05$ was considered statistically significant for all tests.

\section{Results \\ Demographic and clinical characteristics}

No significant differences were observed between MDD patients and $\mathrm{HC}$ groups in the demographics, including age 
Table 2 Comparison of general characteristics of patients with depression and $\mathrm{HCs}$

\begin{tabular}{lllll}
\hline Characteristics & MDD & HC & t & P-value \\
\hline Gender (male/female) & $22 / 40$ & $21 / 52$ & - & $0.460^{\mathrm{a}}$ \\
Age (years) & $28.35 \pm 8.54$ & $27.67 \pm 7.80$ & -0.486 & $0.628^{\mathrm{b}}$ \\
Years of education (years) & $15.56 \pm 3.08$ & $14.58 \pm 3.84$ & 1.688 & $0.098^{\mathrm{b}}$ \\
HAMD score & $22.74 \pm 4.877$ & - & - & - \\
HAMA score & $17.51 \pm 5.778$ & - & - & - \\
\hline
\end{tabular}

Notes: ${ }^{a} P$-value for gender distribution was obtained by chi-square test. ${ }^{b} P$-values were obtained by Student's $t$-test.

Abbreviations: MDD, major depressive disorder; HC, healthy control; HAMD, Hamilton Depression Rating Scale; HAMA, Hamilton Anxiety Rating Scale.

(MDD: 28.35 $\pm 8.54 ; \mathrm{HC}: 27.67 \pm 7.80 ; t=-0.486 ; P=0.628)$, gender (male/female; MDD: 22/40; HC: 21/52; $P=0.460$ ), or education years (MDD: 15.56 \pm 3.08 ; HC: $14.58 \pm 3.84$; $t=1.688 ; P=0.098$ ). The mean severity of clinical symptoms in MDD patients was $22.74 \pm 4.877$ in HAMD and 17.51 \pm 5.778 in HAMA (Table 2).

\section{MicroR-I 32 expression level}

We found that miR-132 expression levels were significantly upregulated in MDD patients compared with HCs $(t=-2.613$; $P=0.01)$. The relative expression of miR-132 between patients and control samples is shown in Figure 1.

\section{Visual memory task results}

Altogether 62 patients and $73 \mathrm{HCs}$ completed all the neurocognitive tests. Between the two groups, we found that MDD patients performed poorer than HCs in PRM - number correct

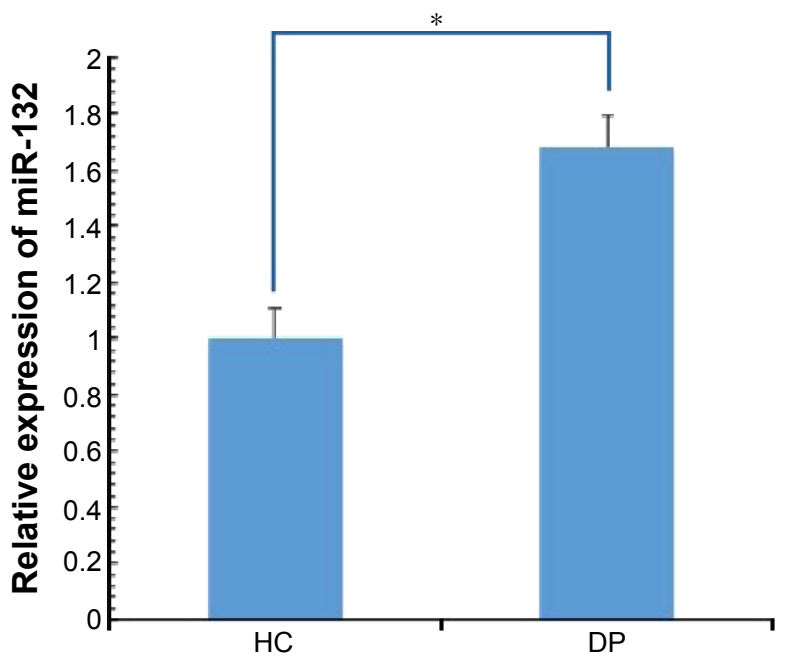

Figure I Blood-based miR-I 32 levels (indicated by $2^{-\Delta \Delta c_{t}}$ ): drug-free DPs vs HCs. Notes: $* P=0.010$. Relative expression of miR- 132 in the two groups is expressed as mean $\pm \mathrm{SE}$ in the bar chart. Differences in $\mathrm{RQ}$ values between the two groups were statistically measured by Student's $t$-test.

Abbreviations: miR-132, microRNA-132; HC, healthy control; DP, depression patient; RQ, relative quantification.
(delayed)(MDD: $9.10 \pm 1.87, \mathrm{HC}: 9.95 \pm 1.75, t=2.722, P=0.007$ ) and PRM - percent correct (delayed) (MDD: 75.84 \pm 15.56 , HC: $82.86 \pm 14.61, t=2.702, P=0.008$; Table 3).

\section{Correlations among miR- 132 expression level, visual memory function, and clinical symptoms}

In the MDD group, the increased miR-132 expression levels were negatively correlated with PRM - number correct (delayed) $(r=-0.273, P=0.032)$ and PRM - percent correct (delayed) $(r=-0.274, P=0.031)$. However, there was no correlation between miR-132 levels and performance in the $\mathrm{HC}$ group. In the MDD group, HAMA scores were negatively correlated with PRM - number correct (immediate) ( $r=-0.301, P=0.017$ ) and PRM - percent correct (immediate) ( $r=-0.305, P=0.016$; Table 4).

No significant relationship between miR-132 level and HAMD $(r=0.019, P=0.883)$ or HAMA $(r=0.175, P=0.172)$ scores in the MDD group was observed.

\section{Discussion}

In our study, we found that visual memory function in MDD patients was significantly decreased compared to that in HCs. MDD patients showed a lower correct number and correct percent than HCs in the delayed modes of PRM. Numerous studies have reported an association between MDD and memory impairments. ${ }^{36,37}$ Using a variety of cognitive tasks, Airaksinen et $\mathrm{al}^{1}$ analyzed the data from patients with major depression, dysthymia, mixed anxiety-depressive disorder, and minor depression. Their results showed that major depression and mixed anxiety-depression disorder groups exhibited significant memory dysfunction, whereas minor depression did not affect cognitive performance. Landrø et $\mathrm{al}^{38}$ reported that patients with MDD performed significantly worse than HCs in working memory and verbal long-term memory. Some studies have found MDD patients were impaired in both verbal memory and visual memory ${ }^{39,40}$ and delayed verbal memory and verbal percent retention, but not in immediate verbal memory or visual memory. ${ }^{37}$ Another study has found that immediate and delayed visual-spatial memories were impaired while immediate and verbal longterm memories were preserved. ${ }^{41}$ Our results showed that delayed visual memory decreased in patients with MDD. This finding was consistent with previous research outcomes.

Studies have revealed that miR-132 expression has tissue specificity, which is highly expressed in nervous tissues and involved in axonal growth, proliferation and differentiation of synapses, and neural tumor formation process. ${ }^{42}$ In addition, 
Table 3 Comparison of the expression of cognition scores between patients with depression and HCs

\begin{tabular}{|c|c|c|c|c|c|}
\hline Test item & Measurement & $\mathrm{HC}$ & MDD & $\boldsymbol{t}$ & $P$-value \\
\hline PRM_NCi & PRM - number correct (immediate) & $11.05 \pm 1.39$ & $11.06 \pm 1.42$ & -0.040 & 0.971 \\
\hline PRM_PCi & PRM - number correct (immediate) & $92.14 \pm 11.68$ & $92.31 \pm 11.90$ & -0.083 & 0.934 \\
\hline PRM_MCLi & PRM - mean correct latency (immediate) & $2,095.48 \pm 896.50$ & $2,3|7.3| \pm|, 5| 0.9 \mid$ & -1.167 & 0.293 \\
\hline PRM_NCd & PRM - number correct (delayed) & $9.95 \pm 1.75$ & $9.10 \pm 1.87$ & 2.722 & $0.007^{*}$ \\
\hline PRM_PCd & PRM - percent correct (delayed) & $82.86 \pm|4.6|$ & $75.84 \pm 15.56$ & 2.702 & $0.008^{*}$ \\
\hline PRM_MCLd & PRM - mean correct latency (delayed) & $2,047.58 \pm 621.16$ & $2,195.68 \pm 757.48$ & -1.255 & 0.245 \\
\hline
\end{tabular}

Notes: Lower scores represent better neuropsychological performance. $* P<0.05$.

Abbreviations: $\mathrm{HC}$, healthy control; MDD, major depressive disorder; PRM, pattern recognition memory.

miR-132 expression levels were the highest among upregulated miRNAs in response to neuronal activity in the dentate gyrus, which was implicated in mood disorders. ${ }^{43}$ One study demonstrated that miR-132 expression in neurons and glia mediated the brain-derived neurotrophic factor (BDNF) action whose low levels are implicated in depression. ${ }^{44}$ Suppression of miR-132 by 2- $O^{\prime}$-methyl oligoribonucleotide can increase BDNF transcript levels. ${ }^{5}$ Studies reported that miR-132 expression was increased in the peripheral blood samples of patients with MDD, while BDNF and MeCP2 were decreased, which suggested that miR-132 may be a key factor in controlling stress-induced hippocampal neuroplasticity and neuronal survival in depression. ${ }^{44,45}$ In line with previous findings, our results confirmed that MDD patients had increased miR-132 expression level.

Numerous behavioral studies in animal models have identified behavioral changes in rodents accompanied with the disruption in the miR-132 expression level. ${ }^{5}$ Hansen et al ${ }^{29}$ demonstrated impaired recognition memory in transgenic mice with overexpressed miR-132 throughout the forebrain. Scott et $a{ }^{46}$ reported that specific overexpression of miR-132 selectively in the perirhinal cortex of rat was associated with impairment of short-term recognition memory. The primary function of miR-132 in response to neural activity is to serve as a proteome switch, broadly modulating the translation of proteins critical for the establishment or maintenance of memory. ${ }^{47}$ Our study found that delayed visual memory deficits were positively correlated with overexpression of miR-132 in DPs. To our knowledge, this is the first study to explore the possible association between alterations in visual memory function of patients with depression and the miR-132 expression level.

Correlation analyses also suggested the association between memory deficits and clinical symptoms in patients with depression (Table 4). In the PRM test, anxiety score and the correct number and the correct percent of pattern recognition were negatively correlated in patients with depression. A number of investigations have indicated that only some depressed individuals have memory dysfunction, but these studies still cannot clearly identify who those individuals are. ${ }^{48,49}$ However, another study suggested that anxious individuals perform less well than non-anxious individuals on

Table 4 Relationship between PRM scores and miR-132 expression and HAMD or HAMA scores in 62 patients and 73 HCs

\begin{tabular}{llll}
\hline & miR-I32 & HAMD & HAMA \\
\hline MDD & & & \\
PRM_NCi & $r=-0.107, P=0.407$ & $r=-0.135, P=0.297$ & $r=-0.30 I, P=0.017^{*}$ \\
PRM_PCi & $r=-0.103, P=0.424$ & $r=-0.135, P=0.295$ & $r=-0.305, P=0.016^{*}$ \\
PRM_MCLi & $r=0.126, P=0.328$ & $r=0.164, P=0.203$ & $r=0.026, P=0.108$ \\
PRM_NCd & $r=-0.273, P=0.032^{*}$ & $r=-0.097, P=0.453$ & $r=-0.101, P=0.437$ \\
PRM_PCd & $r=-0.274, P=0.03 I^{*}$ & $r=-0.100, P=0.440$ & $r=-0.100, P=0.440$ \\
PRM_MCLd & $r=0.139, P=0.283$ & $r=0.288, P=0.230$ & $r=0.113, P=0.383$ \\
HC & & & \\
PRM_NCi & $r=-0.104, P=0.382$ & & \\
PRM_PCi & $r=-0.102, P=0.391$ & & \\
PRM_MCLi & $r=0.006, P=0.959$ & & \\
PRM_NCd & $r=-0.035, P=0.768$ & & \\
PRM_PCd & $r=-0.036, P=0.760$ & & \\
PRM_MCLd & $r=0.042, P=0.721$ & & \\
\hline
\end{tabular}

Note: $* P<0.05$.

Abbreviations: PRM, pattern recognition memory; miR-132, microRNA-132; HAMD, Hamilton Depression Rating Scale; HAMA, Hamilton Anxiety Rating Scale; $\mathrm{HC}$, healthy control; MDD, major depressive disorder. 
memory tasks. ${ }^{50}$ Epidemiological studies from 1972 to 1985 have revealed comorbidity rates of $21 \%-91 \%$ between anxiety and depression. ${ }^{51,52}$ This linkage indicated that depressed individuals may differ and therefore potentially add to the variability of memory functioning. A previous study suggested that among individuals with symptoms of depression and/or anxiety, those experiencing high levels of coexisting depression and anxiety are more likely to have immediate and delayed memory difficulties than individuals reporting either depressive or anxious symptoms alone or those with low levels of both. ${ }^{53}$ In our study, we found that depressed individuals with anxiety symptoms were positively correlated with immediate visual memory defects.

The current study has three limitations. First, the sample size was relatively small. Some patients did not finish the PRM task, which may affect the results of cognition analysis. Second, our MDD patients were not followed over time, and for this reason, it was difficult for us to determine the alteration of miR-132 expression in depression symptoms' recovery or memory improvement. We yet cannot determine whether the reported relation between miRNA-132 alteration and visual memory impairment was restricted to periods of acute episode or persisted during recovery. Finally, we need further experiments to explore the causal relationship between upregulated miR-132 expression level and visual memory changes.

\section{Conclusion}

We found that: 1) miR-132 expression levels were upregulated in major depression; 2) peripheral miR-132 expression levels were associated with visual memory defects in patients with depression; and 3) visual memory dysfunction was positively correlated with clinical symptoms.

\section{Acknowledgment}

This research was partly supported by the National High Technology Research and Development Program of China (863 Program, No 2015AA020513), National Science and Technology Program (2015BAI13B02), and National Natural Science Foundation of China (91232711).

\section{Disclosure}

The authors report no conflicts of interest in this work.

\section{References}

1. Airaksinen E, Larsson M, Lundberg I, Forsell Y. Cognitive functions in depressive disorders: evidence from a population-based study. Psychol Med. 2004;34(1):83-91.

2. Mueller TI, Leon AC, Keller MB, et al. Recurrence after recovery from major depressive disorder during 15 years of observational follow-up. Am J Psychiatry. 1999;156(7):1000-1006.
3. Austin M-P, Mitchell P, Goodwin GM. Cognitive deficits in depression possible implications for functional neuropathology. Br J Psychiatry. 2001:178(3):200-206.

4. Castaneda AE, Tuulio-Henriksson A, Marttunen M, Suvisaari J, Lönnqvist J. A review on cognitive impairments in depressive and anxiety disorders with a focus on young adults. $J$ Affect Disord. 2008; 106(1):1-27.

5. Griffiths S, Scott H, Glover C, et al. Expression of long-term depression underlies visual recognition memory. Neuron. 2008;58(2):186-194.

6. OP, Waterreus A, Spry N, Flicker L, Martins RN. One year follow-up study of the association between chemical castration, sex hormones, beta-amyloid, memory and depression in men. Psychoneuroendocrinology. 2004;29(8):1071-1081.

7. Hickie I, Naismith S, Ward PB, et al. Reduced hippocampal volumes and memory loss in patients with early- and late-onset depression. Br J Psychiatry. 2005;186:197-202.

8. Jirtle RL, Skinner MK. Environmental epigenomics and disease susceptibility. Nat Rev Genet. 2007;8(4):253-262.

9. Cortijo S, Wardenaar R, Colomé-Tatché M, et al. Mapping the epigenetic basis of complex traits. Science. 2014;343(6175):1145-1148.

10. Mill J, Petronis A. Molecular studies of major depressive disorder: the epigenetic perspective. Mol Psychiatry. 2007;12(9):799-814.

11. Huntzinger E, Izaurralde E. Gene silencing by microRNAs: contributions of translational repression and mRNA decay. Nat Rev Genet. 2011;12(2):99-110.

12. Bartel DP. MicroRNAs: target recognition and regulatory functions. Cell. 2009;136(2):215-233.

13. Eulalio A, Huntzinger E, Izaurralde E. Getting to the root of miRNAmediated gene silencing. Cell. 2008;132(1):9-14.

14. Kozomara A, Griffiths-Jones S. miRBase: integrating microRNA annotation and deep-sequencing data. Nucleic Acids Res. 2011;39 (Database issue):D152-D157.

15. Krol J, Loedige I, Filipowicz W. The widespread regulation of microRNA biogenesis, function and decay. Nat Rev Genet. 2010;11(9):597.

16. Konopka W, Kiryk A, Novak M, et al. MicroRNA loss enhances learning and memory in mice. J Neurosci. 2010;30(44):14835-14842.

17. Gardiner E, Beveridge NJ, Wu JQ, et al. Imprinted DLK1-DIO3 region of 14q32 defines a schizophrenia-associated miRNA signature in peripheral blood mononuclear cells. Mol Psychiatry. 2012;17(8):827-840.

18. Katsuura S, Kuwano Y, Yamagishi N, et al. MicroRNAs miR144/144* and miR-16 in peripheral blood are potential biomarkers for naturalistic stress in healthy Japanese medical students. Neurosci Lett. 2012;516(1):79-84.

19. Lai CY, Yu SL, Hsieh MH, et al. MicroRNA expression aberration as potential peripheral blood biomarkers for schizophrenia. PLoS One. 2011;6(6):e21635.

20. Rong H, Liu TB, Yang KJ, et al. MicroRNA-134 plasma levels before and after treatment for bipolar mania. J Psychiatr Res. 2011;45(1):92-95.

21. Hébert SS, Papadopoulou AS, Smith P, et al. Genetic ablation of Dicer in adult forebrain neurons results in abnormal tau hyperphosphorylation and neurodegeneration. Hum Mol Genet. 2010;19(20): 3959-3969.

22. Lee S-T, Chu K, Im W-S, et al. Altered microRNA regulation in Huntington's disease models. Exp Neurol. 2011;227(1):172-179.

23. Johnson R, Zuccato C, Belyaev ND, Guest DJ, Cattaneo E, Buckley NJ. A microRNA-based gene dysregulation pathway in Huntington's disease. Neurobiol Dis. 2008;29(3):438-445.

24. Hollander JA, Im H-I, Amelio AL, et al. Striatal microRNA controls cocaine intake through CREB signalling. Nature. 2010;466(7303): 197-202.

25. Mouillet-Richard S, Baudry A, Launay J-M, Kellermann O. MicroRNAs and depression. Neurobiol Dis. 2012;46(2):272-278.

26. Alvarez-Saavedra M, Antoun G, Yanagiya A, et al. miRNA-132 orchestrates chromatin remodeling and translational control of the circadian clock. Hum Mol Genet. 2010;20(4):731-751.

27. Vo N, Klein ME, Varlamova O, et al. A cAMP-response element binding protein-induced microRNA regulates neuronal morphogenesis. Proc Natl Acad Sci U S A. 2005;102(45):16426-16431. 
28. Impey S, Davare M, Lasiek A, et al. An activity-induced microRNA controls dendritic spine formation by regulating Rac1-PAK signaling. Mol Cell Neurosci. 2010;43(1):146-156.

29. Hansen KF, Sakamoto K, Wayman GA, Impey S, Obrietan K. Transgenic miR132 alters neuronal spine density and impairs novel object recognition memory. PLoS One. 2010;5(11):e15497.

30. Edbauer D, Neilson JR, Foster KA, et al. Regulation of synaptic structure and function by FMRP-associated microRNAs miR-125b and miR-132. Neuron. 2010;65(3):373-384.

31. First MB, Spitzer RL, Gibbon M, Williams JB. User's Guide for the Structured Clinical Interview for DSM-IV Axis I Disorders SCID-I: Clinician Version. Washington, DC: American Psychiatric Pub; 1997.

32. APA. Diagnostic and Statistical Manual of Mental Disorders: DSM-5. Washington, DC: APA; 2003.

33. Shang C, Gau S. Visual memory as a potential cognitive endophenotype of attention deficit hyperactivity disorder. Psychol Med. 2011;41(12): 2603-2614.

34. Barrett SL, Mulholland CC, Cooper SJ, Rushe TM. Patterns of neurocognitive impairment in first-episode bipolar disorder and schizophrenia. Br J Psychiatry. 2009;195(1):67-72.

35. Sahakian BJ, Morris RG, Evenden JL, et al. A comparative study of visuospatial memory and learning in Alzheimer-type dementia and Parkinson's disease. Brain. 1988;111(3):695-718.

36. Campbell S, MacQueen G. The role of the hippocampus in the pathophysiology of major depression. J Psychiatry Neurosci. 2004; 29(6):417.

37. Vythilingam M, Vermetten E, Anderson GM, et al. Hippocampal volume, memory, and cortisol status in major depressive disorder: effects of treatment. Biol Psychiatry. 2004;56(2):101-112.

38. Landrø NI, Stiles TC, Sletvold H. Neuropsychological function in nonpsychotic unipolar major depression. Neuropsychiatry Neuropsychol Behav Neurol. 2001;14(4):233-240.

39. Reischies FM, Neu P. Comorbidity of mild cognitive disorder and depression-a neuropsychological analysis. Eur Arch Psychiatry Clin Neurosci. 2000;250(4):186-193.

40. Naismith SL, Hickie IB, Turner K, et al. Neuropsychological performance in patients with depression is associated with clinical, etiological and genetic risk factors. J Clin Exp Neuropsychol. 2003; 25(6):866-877.
41. Porter RJ, Gallagher P, Thompson JM, Young AH. Neurocognitive impairment in drug-free patients with major depressive disorder. $\mathrm{Br} J$ Psychiatry. 2003;182(3):214-220.

42. Wanet A, Tacheny A, Arnould T, Renard P. miR-212/132 expression and functions: within and beyond the neuronal compartment. Nucleic Acids Res. 2012;40(11):4742-4753.

43. Luikart BW, Perederiy JV, Westbrook GL. Dentate gyrus neurogenesis, integration and microRNAs. Behav Brain Res. 2012;227(2):348-355.

44. Numakawa T, Yamamoto N, Chiba S, et al. Growth factors stimulate expression of neuronal and glial miR-132. Neurosci Lett. 2011;505(3): 242-247.

45. Su M, Hong J, Zhao Y, Liu S, Xue X. MeCP2 controls hippocampal brain-derived neurotrophic factor expression via homeostatic interactions with microRNA-132 in rats with depression. Mol Med Rep. 2015;12(4):5399-5406.

46. Scott HL, Tamagnini F, Narduzzo KE, et al. MicroRNA-132 regulates recognition memory and synaptic plasticity in the perirhinal cortex. Eur J Neurosci. 2012;36(7):2941-2948.

47. Bredy TW, Lin Q, Wei W, Baker-Andresen D, Mattick JS. MicroRNA regulation of neural plasticity and memory. Neurobiol Learn Mem. 2011;96(1):89-94.

48. Burt DB, Zembar MJ, Niederehe G. Depression and memory impairment: a meta-analysis of the association, its pattern, and specificity. Psychol Bull. 1995;117(2):285.

49. Veiel HO. A preliminary profile of neuropsychological deficits associated with major depression. J Clin Exp Neuropsychol. 1997;19(4): 587-603.

50. Eysenck MW, Calvo MG. Anxiety and performance: the processing efficiency theory. Cogn Emot. 1992;6(6):409-434.

51. Kessler RC, Nelson CB, McGonagle KA, Liu J. Comorbidity of DSM-III - R major depressive disorder in the general population: results from the US National Comorbidity Survey. Br J Psychiatry. 1996; (30):17-30.

52. Zajecka JM, Ross JS. Management of comorbid anxiety and depression. J Clin Psychiatry. 1995;56(suppl 2):10-13.

53. Kizilbash AH, Vanderploeg RD, Curtiss G. The effects of depression and anxiety on memory performance. Arch Clin Neuropsychol. 2002; 17(1):57-67.
Neuropsychiatric Disease and Treatment

\section{Publish your work in this journal}

Neuropsychiatric Disease and Treatment is an international, peerreviewed journal of clinical therapeutics and pharmacology focusing on concise rapid reporting of clinical or pre-clinical studies on a range of neuropsychiatric and neurological disorders. This journa is indexed on PubMed Central, the 'PsycINFO' database and CAS,

\section{Dovepress}

and is the official journal of The International Neuropsychiatric Association (INA). The manuscript management system is completely online and includes a very quick and fair peer-review system, which is all easy to use. Visit http://www.dovepress.com/testimonials.php to read real quotes from published authors. 